\title{
RECURSIVE MAXIMUM LIKELIHOOD ESTIMATION FOR HIDDEN SEMI-MARKOV MODELS
}

\author{
Kevin Squire and Stephen Levinson \\ University of Illinois at Urbana-Champaign \\ Department of Electrical and Computer Engineering \\ Beckman Institute, 405 N. Mathews, Urbana, IL 61801
}

\begin{abstract}
The term hidden semi-Markov model (HSMM) refers to a large class of stochastic models developed to address some of the shortcomings of hidden Markov models (HMMs). As with HMMs, the underlying sequence of states of a process is modelled as a discrete Markov chain. Unlike HMMs, each state in an HSMM can emit a variable length sequence of observations, with many ways to model duration and observation densities. Parameter estimation in HSMMs is typically done using EM or Viterbi (dynamic programming) algorithms. In this paper, we present a recursive maximumlikelihood estimation (RMLE) algorithm for HSMM parameter estimation, based on a similar method developed for HMMs [1,2].
\end{abstract}

\section{INTRODUCTION}

When applying HMMs to speech and other continuous data, a general assumption is that each state in the model represents a stationary interval over a data segment. With a standard HMM, the probability of duration of a state is implicitly modeled as a geometric distribution, which does not accurately model the temporal structure of speech or many other processes. To address this problem, Fergusson [3] proposed the idea of a variable duration hidden Markov model, which explicitly models the duration of a given state with a probability mass function and converts the underlying Markov chain to a semi-Markov chain. Russell and Moore [4] and Levinson [5] extended this work by modeling the state duration with Poisson and gamma distributions, respectively. Various other researchers have extended this idea or independently developed related ideas, producing a variety of models that have variously been called hidden semi-Markov models [6], variable-duration hidden Markov models [3-5, 7], generalized HMMs [8], segmental HMMs [9], and segment models [10]. In this paper, we have somewhat arbitrarily chosen to refer to this class of models as hidden semi-Markov models, or HSMMs. (See [10] for a unifying overview and survey of most of these models.)
As with HMMs, EM or Viterbi (dynamic programming) algorithms are typically used for parameter estimation in HSMMs. Recently, there has been interest in developing recursive algorithms for HMM parameter estimation [1, 2, 11-15]. The work presented here generalizes the recursive maximum-likelihood estimation (RMLE) algorithm developed for HMMs to HSMMs. In this paper we adopt the much of the notation and form of a similar method presented in [2], which is based on earlier work by [1].

In the sections that follow, we will describe the mathematical model for the HSMM, and then give a derivation of the RMLE for this model.

\section{HSMM MODEL DESCRIPTION AND NOTATION}

In the discussion that follows, the model signal will be analyzed on two different time scales, which we will refer to as model time and normal time. For model-time analysis, time variables are marked with a prime $\left({ }^{\prime}\right)$, and sequence variables are marked with an overbar, as in $\bar{\tau}_{n^{\prime}}$. For normaltime analysis, the prime and overbar will be absent, as in $\tau_{n}$. The relationship between model time and normal time will be described later.

An HSMM is a discrete-time stochastic process with three components, $\left\{\bar{X}_{n^{\prime}}, \overline{\mathcal{T}}_{n^{\prime}}, \bar{Y}_{n^{\prime}}\right\}$, defined on probability space $(\Omega, \mathcal{F}, P)$. Let $\left\{\bar{X}_{n^{\prime}}\right\}_{n^{\prime}=1}^{\infty}$ be a discrete-time firstorder Markov chain with state space $R=\{1, \ldots, r\}, r$ a fixed known constant. The model starts in a particular state $i=1, \ldots, r$ with probability $\pi_{i}=P\left(X_{1}=i\right)$. Define $\boldsymbol{\pi} \in \Pi$ by $\boldsymbol{\pi}=\left\{\pi_{i}\right\}$, where $\Pi$ is the set of length- $r$ stochastic vectors. The transition probabilities of the Markov chain are given by

$$
a_{i j}=P\left(\bar{X}_{n^{\prime}}=j \mid \bar{X}_{n^{\prime}-1}=i\right)
$$

for $i, j=1, \ldots, r$, with an additional constraint that $a_{i i}=$ $P\left(\bar{X}_{n^{\prime}}=i \mid \bar{X}_{n^{\prime}-1}=i\right)=0$. Let $\mathbf{A}=\left\{a_{i j}\right\}$. Then $\mathbf{A} \in \mathcal{A} \backslash\left\{a_{i i} \neq 0\right\}$, where $\mathcal{A}$ is the set of all $r \times r$ stochastic matrices (i.e., $a_{i j} \geq 0, \sum_{j} a_{i j}=1$ ).

$\overline{\mathcal{T}}_{k^{\prime}}$ is the discrete, normal-time duration the process remains in state $\bar{X}_{k^{\prime}}$ at model-time $k^{\prime}$. Let $\left\{\overline{\mathcal{T}}_{n^{\prime}}\right\}$ be a proba- 
bilistic function of $\left\{\bar{X}_{n^{\prime}}\right\}$, and let the conditional density of $\overline{\mathcal{T}}_{n^{\prime}}$ be described by a parametric family of densities $\{d(\cdot ; \lambda): \lambda \in \Lambda\}$, where the density parameter $\lambda$ is a function of $\bar{X}_{n^{\prime}}$, and $\Lambda$ is the set of valid parameters for the conditional density assumed by the model. The conditional density of $\overline{\mathcal{T}}_{n^{\prime}}$ given $\bar{X}_{n^{\prime}}=j$ can be written $d\left(\cdot ; \lambda_{j}\right)$, or more simply $d_{j}(\cdot)$. (More complex time duration models are possible [10]. Note, however, that the conditional independence of the duration given the current state is required for the algorithm described here.)

Example 1 (Gamma duration density): Suppose the durations for each state in an HMM are approximately ${ }^{1}$ distributed according to a Gamma distribution. Then parameter set $\Lambda=\left\{(\nu, \eta) \in \mathbb{R}^{+} \times \mathbb{R}^{+}\right\}, \lambda_{j} \in \Lambda$, and $\left\{\overline{\mathcal{T}}_{n^{\prime}}\right\}=$ $\left\{\bar{\tau}_{n^{\prime}}\right\}$ is a sequence of discrete valued conditionally independent state durations on $\mathbb{R}^{+}$, with probability distribution

$$
d\left(\bar{\tau}_{n^{\prime}} ; \lambda_{j}\right)=d\left(\bar{\tau}_{n^{\prime}} ; \nu_{j}, \eta_{j}\right)=\frac{\eta_{j}^{\nu_{j}}}{\Gamma\left(\nu_{j}\right)} \bar{\tau}_{n^{\prime}}^{\nu_{j}-1} e^{-\eta_{j} \bar{\tau}_{n^{\prime}}}
$$

for $X_{n}=j$. Here, the mean value of $\bar{\tau}_{n}$ is $\frac{\nu_{j}}{\eta_{j}}$, and the variance is $\frac{\nu_{j}}{\eta_{j}^{2}}$.

Example 2 (Discrete duration density): Suppose durations $\left\{\overline{\mathcal{T}}_{n^{\prime}}\right\}$ are drawn from a discrete set of times $\mathfrak{T}=\left\{t_{1}, \ldots, t_{T}\right\}$. Then $\Lambda=\left\{\left(d_{1}, \ldots, d_{T}\right) \mid \sum_{\tau=1}^{T} d_{\tau}=\right.$ $\left.1, d_{\tau} \geq 0\right\}$ is the set of length-T stochastic vectors, $\lambda_{j} \in \Lambda$, and $\left\{\overline{\mathcal{T}}_{n^{\prime}}\right\}=\left\{\bar{\tau}_{n^{\prime}}\right\}$ is a sequence of discrete valued conditionally independent state durations on $\mathfrak{T}$, each $\bar{\tau}_{n}$ having probability

$$
d\left(\bar{\tau}_{n^{\prime}} ; \lambda_{j}\right)=d_{j \bar{\tau}_{n^{\prime}}} \quad 1 \leq \bar{\tau}_{n^{\prime}} \leq T
$$

for $\bar{X}_{n^{\prime}}=j$.

In an HSMM, state $\bar{X}_{n^{\prime}}$ produces a length $\overline{\mathcal{T}}_{n^{\prime}}$ observation vector $\bar{Y}_{n^{\prime}}$. The process $\left\{\bar{Y}_{n^{\prime}}\right\}$ therefore is a probabilistic function of $\left\{\bar{X}_{n^{\prime}}\right\}$ and $\left\{\overline{\mathcal{T}}_{n^{\prime}}\right\}$, and the corresponding conditional density of $\bar{Y}_{n^{\prime}}$ is assumed to belong to a parametric family of densities $\{b(\cdot \mid \bar{\tau} ; \theta): \theta \in \Theta\}$, where $\bar{\tau}$ is a sample from duration process $\left\{\overline{\mathcal{T}}_{n^{\prime}}\right\}$, the density parameter $\theta$ is a function of $\bar{X}_{n^{\prime}}$, and $\Theta$ is the set of valid parameters for the particular conditional density assumed by the model. The conditional density of $\bar{Y}_{n^{\prime}}$ given $\bar{X}_{n^{\prime}}=j$ and $\overline{\mathcal{T}}_{n^{\prime}}=\bar{\tau}_{n^{\prime}}$ can be written $b\left(\cdot \mid \bar{\tau}_{n^{\prime}} ; \theta_{j}\right)$, or more simply as $b_{j}\left(\cdot \mid \bar{\tau}_{n^{\prime}}\right)$. Many different formulations of this observation density are possible [10]. With the exception of a few conditions outlined below, the particular form of $b\left(\cdot \mid \bar{\tau}_{n^{\prime}} ; \theta_{j}\right)$ is irrelevant to our discussion.

Define the HSMM parameter space as $\Phi=\Pi \times \mathcal{A} \times$ $\Lambda \times \Theta$. The model $\varphi \in \Phi$ is defined as

$$
\boldsymbol{\varphi}=\left\{\pi_{1}, \ldots, \pi_{r}, a_{11}, \ldots, a_{r r}, \lambda_{1}, \ldots, \lambda_{r}, \theta_{1}, \ldots, \theta_{r}\right\} .
$$

\footnotetext{
${ }^{1}$ Since the durations are discrete, the correspondence will not be exact.
}

We will often not be concerned with estimating $\pi$; in these cases, we will drop it from the model definition.

Example 3 (Gamma duration densities with Gaussian observation densities): For the case of gamma duration densities with single dimensional Gaussian observation distributions, and ignoring initial state probabilities,

$$
\boldsymbol{\varphi}=\left(a_{11}, \ldots, a_{r r}, \nu_{1}, \eta_{1}, \ldots, \nu_{r}, \eta_{r}, \mu_{1}, \sigma_{1}, \ldots, \mu_{r}, \sigma_{r}\right) \text {. }
$$

Let $p$ be the length of $\varphi$. Let $\varphi^{*} \in \Phi$ be the fixed set of true parameters of the model we are trying to estimate.

For a vector or matrix $\mathbf{v}, \mathbf{v}^{\prime}$ represents its transpose. Define the $r$-dimensional column vector $\mathbf{d}\left(\bar{\tau}_{n^{\prime}} ; \varphi\right)$ and $r \times r$ matrix $\mathbf{D}\left(\bar{\tau}_{n^{\prime}} ; \varphi\right)$ by

$$
\mathbf{d}\left(\bar{\tau}_{n^{\prime}} ; \boldsymbol{\varphi}\right)=\left[d\left(\bar{\tau}_{n^{\prime}} ; \lambda_{1}(\boldsymbol{\varphi})\right), \ldots, d\left(\bar{\tau}_{n^{\prime}} ; \lambda_{r}(\boldsymbol{\varphi})\right)\right]^{\prime}
$$

and

$$
\mathbf{D}\left(\bar{\tau}_{n^{\prime}} ; \boldsymbol{\varphi}\right)=\operatorname{diag}\left[\mathbf{d}\left(\bar{\tau}_{n^{\prime}} ; \varphi\right)\right] .
$$

Similarly, define the $r$-dimensional column vector $\mathbf{b}\left(\bar{y}_{n^{\prime}} \mid \bar{\tau}_{n^{\prime}} ; \boldsymbol{\varphi}\right)$ and $r \times r$ matrix $\mathbf{B}\left(\bar{y}_{n^{\prime}} \mid \bar{\tau}_{n^{\prime}} ; \boldsymbol{\varphi}\right)$ by

$$
\mathbf{b}\left(\bar{y}_{n^{\prime}} \mid \bar{\tau}_{n^{\prime}} ; \boldsymbol{\varphi}\right)=\left[b\left(\bar{y}_{n^{\prime}} ; \theta_{1 \bar{\tau}_{n^{\prime}}}(\boldsymbol{\varphi})\right), \ldots, b\left(\bar{y}_{n^{\prime}} ; \theta_{r \bar{\tau}_{n^{\prime}}}(\boldsymbol{\varphi})\right)\right]^{\prime}
$$

and

$$
\mathbf{B}\left(\bar{y}_{n^{\prime}} \mid \bar{\tau}_{n^{\prime}} ; \boldsymbol{\varphi}\right)=\operatorname{diag}\left[\mathbf{b}\left(\bar{y}_{n^{\prime}} \mid \bar{\tau}_{n^{\prime}} ; \boldsymbol{\varphi}\right)\right] .
$$

For convenience of notation, we will define a third vector $\mathbf{g}\left(\bar{y}_{n^{\prime}}, \bar{\tau}_{n^{\prime}} ; \boldsymbol{\varphi}\right)$ and matrix $\mathbf{G}\left(\bar{y}_{n^{\prime}}, \bar{\tau}_{n^{\prime}} ; \boldsymbol{\varphi}\right)$ as

$$
\begin{aligned}
\mathbf{g}\left(\bar{y}_{n^{\prime}}, \bar{\tau}_{n^{\prime}} ; \boldsymbol{\varphi}\right) & =\mathbf{B}\left(\bar{y}_{n^{\prime}} \mid \bar{\tau}_{n^{\prime}} ; \boldsymbol{\varphi}\right) \mathbf{D}\left(\bar{\tau}_{n^{\prime}} ; \boldsymbol{\varphi}\right) \mathbf{1}_{r} \\
& =\mathbf{B}\left(\bar{y}_{n^{\prime}} \mid \bar{\tau}_{n^{\prime}} ; \boldsymbol{\varphi}\right) \mathbf{d}\left(\bar{\tau}_{n^{\prime}} ; \boldsymbol{\varphi}\right)
\end{aligned}
$$

and

$$
\mathbf{G}\left(\bar{y}_{n^{\prime}}, \bar{\tau}_{n^{\prime}} ; \boldsymbol{\varphi}\right)=\mathbf{B}\left(\bar{y}_{n^{\prime}} \mid \bar{\tau}_{n^{\prime}} ; \boldsymbol{\varphi}\right) \mathbf{D}\left(\bar{\tau}_{n^{\prime}} ; \boldsymbol{\varphi}\right) .
$$

These will be used below to define the likelihood of an observation sequence.

Until now, we have described the model entirely using model time, where one time unit corresponds to the duration the model stays in a particular state. We would like to relate this description to normal time, where each time unit represents one real unit of time.

Using notation from [6], for a given sequence of durations $\left\{\bar{\tau}_{n^{\prime}}\right\}$, define the functions $t_{0}: \mathbb{Z}^{+} \rightarrow \mathbb{Z}^{+}$and $t_{1}: \mathbb{Z}^{+} \rightarrow \mathbb{Z}^{+}$by

$$
\begin{aligned}
& t_{0\left\{\bar{\tau}_{n^{\prime}}\right\}}\left(k^{\prime}\right)=\sum_{i=1}^{k^{\prime}-1} \bar{\tau}_{i}+1 \\
& t_{1\left\{\bar{\tau}_{n^{\prime}}\right\}}\left(k^{\prime}\right)=\sum_{i=1}^{k^{\prime}} \bar{\tau}_{i} .
\end{aligned}
$$


These functions mark, respectively, the real beginning and end of the $k^{\text {th }}$ state for duration sequence $\left\{\bar{\tau}_{n^{\prime}}\right\}$.

Similarly, define a function $\xi: \mathbb{Z}^{+} \rightarrow \mathbb{Z}^{+}$as

$$
\xi_{\left\{\bar{\tau}_{n^{\prime}}\right\}}(n)=k^{\prime} \quad \text { if } t_{0\left\{\bar{\tau}_{n^{\prime}}\right\}}\left(k^{\prime}\right) \leq n \leq t_{1\left\{\bar{\tau}_{n^{\prime}}\right\}}\left(k^{\prime}\right) \text {. }
$$

This function provides the model time corresponding to normal-time $n$ for duration sequence $\left\{\bar{\tau}_{n^{\prime}}\right\}$. Together, these functions allow us to convert between the two time scales. When obvious, we will drop the explicit dependence on $\left\{\bar{\tau}_{n^{\prime}}\right\}$ and simply write $t_{0}\left(n^{\prime}\right), t_{1}\left(n^{\prime}\right)$, and $\xi(n)$.

Using these functions, we can define real-time correspondence from $\left\{X_{n}\right\}$ and $\left\{Y_{n}\right\}$ to $\left\{\bar{X}_{n^{\prime}}\right\}$ and $\left\{\bar{Y}_{n^{\prime}}\right\}$, respectively. The process $\left\{X_{n}\right\}$ is related to $\left\{\bar{X}_{n^{\prime}}\right\}$ by $X_{n}=$ $\bar{X}_{\xi_{\left\{\bar{\tau}_{n^{\prime}}\right\}}(n)}$. Random sample $\bar{Y}_{n^{\prime}}$ can be written as $\bar{Y}_{n^{\prime}}=$ $\left\langle Y_{t_{o}\left(n^{\prime}\right)}, \ldots, Y_{t_{1}\left(n^{\prime}\right)}\right\rangle$.

For model $\varphi$, we would like to calculate the likelihood of a sequence of $n$ normal-time observations $\left\langle y_{1}, \ldots, y_{n}\right\rangle$. Since our model is defined in terms of $\left\{\bar{y}_{n^{\prime}}\right\}$, we partition the sequence $\left\{y_{n}\right\}$ into $n^{\prime} \leq n$ subsequences such that each subsequence corresponds to the output of a single state of the model, i.e.,

$$
\underbrace{y_{1}, \ldots, y_{t_{1}(1)}}_{\bar{y}_{1}}, \underbrace{y_{t_{0}(2)}, \ldots, y_{t_{1}(2)}}_{\bar{y}_{2}}, \ldots, \underbrace{y_{t_{0}\left(n^{\prime}\right)}, \ldots, y_{n}}_{\bar{y}_{n^{\prime}}} .
$$

For a given partition, the joint likelihood of the observation sequence and state durations is given by

$$
\begin{gathered}
\bar{p}_{n^{\prime}}\left(\bar{y}_{1}, \ldots, \bar{y}_{n^{\prime}}, \bar{\tau}_{1}, \ldots, \bar{\tau}_{n^{\prime}} ; \boldsymbol{\varphi}\right) \\
=\pi(\boldsymbol{\varphi})^{\prime} \mathbf{D}\left(\bar{\tau}_{1} ; \boldsymbol{\varphi}\right) \mathbf{B}\left(\bar{y}_{1} \mid \bar{\tau}_{1} ; \boldsymbol{\varphi}\right) \\
\times \prod_{k^{\prime}=2}^{n^{\prime}} \mathbf{A}(\boldsymbol{\varphi}) \mathbf{D}\left(\bar{\tau}_{k^{\prime}} ; \boldsymbol{\varphi}\right) \mathbf{B}\left(\bar{y}_{k^{\prime}} \mid \bar{\tau}_{k^{\prime}} ; \boldsymbol{\varphi}\right) \mathbf{1}_{r} \\
=\pi(\boldsymbol{\varphi})^{\prime} \mathbf{G}\left(\bar{y}_{1}, \bar{\tau}_{1} ; \boldsymbol{\varphi}\right) \prod_{k^{\prime}=2}^{n^{\prime}} \mathbf{A}(\boldsymbol{\varphi}) \mathbf{G}\left(\bar{y}_{k^{\prime}}, \bar{\tau}_{k^{\prime}} ; \boldsymbol{\varphi}\right) \mathbf{1}_{r}
\end{gathered}
$$

Averaging over all possible partitions, we can calculate $p_{n}\left(y_{1}, \ldots, y_{n} ; \boldsymbol{\varphi}\right)$ as

$$
\begin{aligned}
& p_{n}\left(y_{1}, \ldots, y_{n} ; \boldsymbol{\varphi}\right) \\
& =\sum_{n^{\prime}=1}^{n} \sum_{\substack{\bar{\tau}_{1}, \ldots, \bar{\tau}_{n}, \sum_{i=1}^{n^{\prime}} \bar{\tau}_{i}=n}} P\left(n^{\prime}\right) \bar{p}_{n^{\prime}}\left(\bar{y}_{1}, \ldots, \bar{y}_{n^{\prime}}, \bar{\tau}_{1}, \ldots, \bar{\tau}_{n^{\prime}} ; \boldsymbol{\varphi}\right) \\
& =\sum_{n^{\prime}=1}^{n} \sum_{\substack{\bar{\tau}_{1}, \ldots, \bar{\tau}_{n}, \sum_{i=1}^{n^{\prime}} \bar{\tau}_{i}=n}} P\left(n^{\prime}\right) \boldsymbol{\pi}(\boldsymbol{\varphi})^{\prime} \mathbf{G}\left(\bar{y}_{1}, \bar{\tau}_{1} ; \boldsymbol{\varphi}\right) \\
& \times \prod_{k^{\prime}=2}^{n^{\prime}} \mathbf{A}(\boldsymbol{\varphi}) \mathbf{G}\left(\bar{y}_{k^{\prime}}, \bar{\tau}_{k^{\prime}} ; \boldsymbol{\varphi}\right) \mathbf{1}_{r}, \quad \text { (15) }
\end{aligned}
$$

where $P\left(n^{\prime}\right)$ is the probability that the partition has $n^{\prime}$ sections.

\section{RMLE FOR THE HSMM}

The derivation of the RMLE algorithm proceeds as follows. We first show how to calculate the likelihood $p_{n}\left(y_{1}, \ldots, y_{n} ; \boldsymbol{\varphi}\right)$ for a given HSMM recursively, using prediction (or forward) filters. We note that maximizing log $p_{n}\left(y_{1}, \ldots, y_{n} ; \boldsymbol{\varphi}\right)$ is equivalent to and generally easier than maximizing $p_{n}\left(y_{1}, \ldots, y_{n} ; \boldsymbol{\varphi}\right)$ [16], and that $\log$ $p_{n}\left(y_{1}, \ldots, y_{n} ; \varphi\right)$ can also be calculated recursively. We can then search for the maximum of $\log p_{n}\left(y_{1}, \ldots, y_{n} ; \boldsymbol{\varphi}\right)$ using the derivative of the update for this recursion.

Define the prediction filter $\overline{\mathbf{u}}_{n^{\prime}}(\boldsymbol{\varphi})$ as

$$
\overline{\mathbf{u}}_{n^{\prime}}(\boldsymbol{\varphi})=\left[\bar{u}_{n^{\prime} 1}(\boldsymbol{\varphi}), \ldots, \bar{u}_{n^{\prime} r}(\boldsymbol{\varphi})\right]^{\prime}
$$

where

$$
\bar{u}_{n^{\prime} j}(\boldsymbol{\varphi})=P\left(\bar{X}_{n^{\prime}}=j \mid \bar{y}_{1}, \ldots, \bar{y}_{n^{\prime}-1}, \bar{\tau}_{1}, \ldots, \bar{\tau}_{n^{\prime}-1}, n^{\prime}\right)
$$

is the probability of transitioning to state $j$ at (model) time $n^{\prime}$ given all previous observations and a partition of those observations. For our derivation below, it will be useful to have a normal-time correlate to $\overline{\mathbf{u}}_{n^{\prime}}(\varphi)$. Let $\mathbf{u}_{n}(\varphi)$ be

$$
\mathbf{u}_{n}(\boldsymbol{\varphi})=\left[u_{n 1}(\boldsymbol{\varphi}), \ldots, u_{n r}(\boldsymbol{\varphi})\right]^{\prime}
$$

where

$$
\begin{array}{r}
u_{n j}(\boldsymbol{\varphi})=P\left(X_{n}=j \mid y_{1}, \ldots, y_{n-1}, \bar{\tau}_{1}, \ldots, \bar{\tau}_{n^{\prime}-1}, n^{\prime}\right. \\
\left.\xi(n-1)=n^{\prime}-1, \xi(n)=n^{\prime}\right) .
\end{array}
$$

For given $n^{\prime}$ and $\left\{\bar{\tau}_{n^{\prime}}\right\}, \overline{\mathbf{u}}_{n^{\prime}}(\boldsymbol{\varphi})=\mathbf{u}_{t_{0}\left(n^{\prime}\right)}(\boldsymbol{\varphi})$.

Using this filter, the likelihood $\bar{p}_{n^{\prime}}$ can be written as

$$
\begin{aligned}
\bar{p}_{n^{\prime}} & \left(\bar{y}_{1}, \ldots, \bar{y}_{n^{\prime}}, \bar{\tau}_{1}, \ldots, \bar{\tau}_{n^{\prime}} ; \boldsymbol{\varphi}\right) \\
= & \prod_{k^{\prime}=1}^{n^{\prime}} \mathbf{d}\left(\bar{\tau}_{k^{\prime}} ; \boldsymbol{\varphi}\right)^{\prime} \mathbf{B}\left(\bar{y}_{k^{\prime}} \mid \bar{\tau}_{k^{\prime}} ; \boldsymbol{\varphi}\right) \overline{\mathbf{u}}_{k^{\prime}}(\boldsymbol{\varphi}) . \\
= & \prod_{k^{\prime}=1}^{n^{\prime}} \mathbf{g}\left(\bar{y}_{k^{\prime}}, \bar{\tau}_{k^{\prime}} ; \boldsymbol{\varphi}\right)^{\prime} \overline{\mathbf{u}}_{k^{\prime}}(\boldsymbol{\varphi}) .
\end{aligned}
$$

(For this derivation, see the Appendix.) As above, the likelihood at (normal-time) $n$ can be calculated by averaging over all partitions of $n$, as

$$
\begin{aligned}
& p_{n}\left(y_{1}, \ldots, y_{n} ; \boldsymbol{\varphi}\right) \\
& =\sum_{\substack{n^{\prime}=1 \\
\sum_{\bar{\tau}}, \ldots, \bar{\tau}_{n^{\prime}} \\
\sum_{i=1}^{n^{\prime}} \bar{\tau}_{i}=n}}^{n} P\left(n^{\prime}\right) \prod_{k^{\prime}=1}^{n^{\prime}} \mathbf{g}\left(\bar{y}_{k^{\prime}}, \bar{\tau}_{k^{\prime}} ; \boldsymbol{\varphi}\right)^{\prime} \overline{\mathbf{u}}_{k^{\prime}}(\boldsymbol{\varphi}) .
\end{aligned}
$$


Our goal is to maximize this likelihood with respect to $\varphi$, and in particular find a recursive update. Unfortunately, there are some pragmatic problems with recursively maximizing Equation (22). In particular, since we would actually like to calculate this likelihood recursively, the summation over all partitions of $\left\langle y_{1}, \ldots, y_{n}\right\rangle$ is undesirable. To alleviate this problem, we will consider only the most likely partition of $\left\langle y_{1}, \ldots, y_{n}\right\rangle$. Rewrite Equation (22) as

$$
\begin{aligned}
& \tilde{p}_{n}\left(y_{1}, \ldots, y_{n} ; \boldsymbol{\varphi}\right) \\
& \quad=\max _{\substack{n^{\prime}=1, . ., n \\
\bar{\tau}_{1}, \ldots, \bar{\tau}_{n^{\prime}} \\
\sum_{i=1}^{n^{\prime}} \bar{\tau}_{i}=n}} \prod_{k^{\prime}=1}^{n^{\prime}} \mathbf{g}\left(\bar{y}_{k^{\prime}}, \bar{\tau}_{k^{\prime}} ; \boldsymbol{\varphi}\right)^{\prime} \overline{\mathbf{u}}_{k^{\prime}}(\boldsymbol{\varphi}) .
\end{aligned}
$$

Instead of maximizing $\tilde{p}_{n}\left(y_{1}, \ldots, y_{n} ; \boldsymbol{\varphi}\right)$, we can equivalently maximize its log-likelihood. For a given partition size $n^{\prime}$ and duration sequence $\left\{\bar{\tau}_{n^{\prime}}\right\}$, define the normalized log-likelihood of (model-time sequence) $\left\langle\bar{y}_{1}, \ldots, \bar{y}_{n^{\prime}}\right\rangle$ as

$$
\begin{aligned}
\bar{\ell}_{n^{\prime}}\left(\left\{\bar{\tau}_{n^{\prime}}\right\}, \boldsymbol{\varphi}\right) & =\frac{1}{n^{\prime}+1} \log \bar{p}_{n^{\prime}}\left(\bar{y}_{1}, \ldots, \bar{y}_{n^{\prime}}, \bar{\tau}_{1}, \ldots, \bar{\tau}_{n^{\prime}} ; \boldsymbol{\varphi}\right) \\
& =\frac{1}{n^{\prime}+1} \sum_{k^{\prime}=1}^{n^{\prime}} \log \mathbf{g}\left(\bar{y}_{k^{\prime}}, \bar{\tau}_{k^{\prime}} ; \boldsymbol{\varphi}\right)^{\prime} \overline{\mathbf{u}}_{k^{\prime}}(\boldsymbol{\varphi})
\end{aligned}
$$

As in Equation (23), we can write the log-likelihood of real time observations $\left\langle y_{1}, \ldots, y_{n}\right\rangle$ as

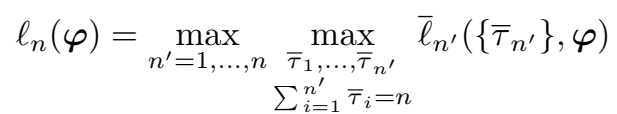

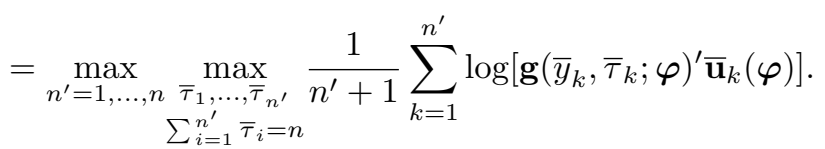

At any time $n$, the values of $n^{\prime}$ and $\left\{\bar{\tau}_{n^{\prime}}\right\}$ which maximize $\ell_{n}(\varphi)$ can be determined recursively through dynamic programming, and can also be used in the recursive update of $\mathbf{u}_{n}(\boldsymbol{\varphi})$. Let $n_{n}^{\prime *}$ be the number of segments which maximizes $\ell_{n}(\varphi)$, and let $\tau_{n}^{*}$ be the length of the last segment of $\left\{\bar{y}_{n^{\prime}}\right\}$ which maximizes $\ell_{n}(\varphi)$. Given the sequence of loglikelihoods up to $\ell_{n-1}(\varphi)$, as well as the optimal state sequence lengths $n_{1}^{* *}$ through $n_{n-1}^{*}$, we can maximize $\ell_{n}(\varphi)$ recursively with

$$
\begin{aligned}
\tau_{n}^{*}= & \underset{\tau}{\arg \max } \frac{1}{n_{n-\tau}^{\prime *}+2}\left(\left(n_{n-\tau}^{\prime *}+1\right) \ell_{n-\tau}(\boldsymbol{\varphi})\right. \\
& \left.+\log \left[\mathbf{g}\left(y_{n-\tau+1}, \ldots, y_{n}, \tau ; \boldsymbol{\varphi}\right)^{\prime} \mathbf{u}_{n-\tau+1}(\boldsymbol{\varphi})\right]\right)
\end{aligned}
$$

$$
n_{n}^{\prime *}=n_{n-\tau_{n}^{*}}^{*}+1
$$

and

$$
\begin{aligned}
\ell_{n}(\boldsymbol{\varphi})= & \max _{\tau} \frac{1}{n_{n-\tau}^{\prime *}+2}\left(\left(n_{n-\tau}^{\prime *}+1\right) \ell_{n-\tau}(\boldsymbol{\varphi})\right. \\
& \left.+\log \left[\mathbf{g}\left(y_{n-\tau+1}, \ldots, y_{n}, \tau ; \boldsymbol{\varphi}\right)^{\prime} \mathbf{u}_{n-\tau+1}(\boldsymbol{\varphi})\right]\right),
\end{aligned}
$$

with initializations $\tau_{1}^{*}=1, n_{1}^{\prime *}=1$, and $\ell_{1}(\varphi)=\frac{1}{2} \log$ $\left[\mathbf{g}\left(y_{1}, \tau_{1}^{*} ; \boldsymbol{\varphi}\right)^{\prime} \mathbf{u}_{1}(\boldsymbol{\varphi})\right]$.

As suggested above, we then use $\tau_{n}^{*}$ to recursively calculate $\mathbf{u}_{n}(\boldsymbol{\varphi})$, using

$$
\begin{aligned}
\mathbf{u}_{n+1}(\boldsymbol{\varphi}) & \\
= & \frac{\mathbf{A}(\boldsymbol{\varphi})^{\prime} \mathbf{G}\left(y_{n-\tau_{n}^{*}+1}, \ldots, y_{n}, \tau_{n}^{*} ; \boldsymbol{\varphi}\right) \mathbf{u}_{n-\tau_{n}^{*}+1}(\boldsymbol{\varphi})}{\mathbf{g}\left(y_{n-\tau_{n}^{*}+1}, \ldots, y_{n}, \tau_{n}^{*} ; \boldsymbol{\varphi}\right)^{\prime} \mathbf{u}_{n-\tau_{n}^{*}+1}(\boldsymbol{\varphi})} \\
= & \frac{\mathbf{A}(\boldsymbol{\varphi})^{\prime} \mathbf{G}\left(\bar{y}_{\xi(n)}, \tau_{n}^{*} ; \boldsymbol{\varphi}\right) \mathbf{u}_{n-\tau_{n}^{*}+1}(\boldsymbol{\varphi})}{\mathbf{g}\left(\bar{y}_{\xi(n)}, \tau_{n}^{*} ; \boldsymbol{\varphi}\right)^{\prime} \mathbf{u}_{n-\tau_{n}^{*}+1}(\boldsymbol{\varphi})}
\end{aligned}
$$

with $\mathbf{u}_{1}(\varphi)$ initialized to $\boldsymbol{\pi}(\boldsymbol{\varphi})$.

Let $\mathbf{w}_{n}^{(l)}(\boldsymbol{\varphi})=\left(\partial / \partial \varphi_{l}\right) \mathbf{u}_{n}(\boldsymbol{\varphi})$ be the partial derivative of $\mathbf{u}_{n}(\varphi)$ with respect to (wrt) the $l$ th component of $\varphi$. Each $\mathbf{w}_{n}^{(l)}(\varphi)$ is an $r$-length column vector, and

$$
\mathbf{w}_{n}(\boldsymbol{\varphi})=\left(\mathbf{w}_{n}^{(1)}(\boldsymbol{\varphi}), \mathbf{w}_{n}^{(2)}(\boldsymbol{\varphi}), \ldots, \mathbf{w}_{n}^{(p)}(\boldsymbol{\varphi})\right)
$$

is an $r \times p$ matrix. Taking the derivative of $\mathbf{u}_{n}(\boldsymbol{\varphi})$ from Equation (29), we get

$$
\begin{aligned}
& \mathbf{w}_{n+1}^{(l)}(\boldsymbol{\varphi})=\frac{\partial \mathbf{u}_{n+1}(\boldsymbol{\varphi})}{\partial \varphi_{l}} \\
& \quad=R_{1}\left(\bar{y}_{\xi(n)}, \tau_{n}^{*}, \boldsymbol{\varphi}\right) \mathbf{w}_{n-\tau_{n}^{*}+1}^{(l)}(\boldsymbol{\varphi})+R_{2}^{(l)}\left(\bar{y}_{\xi(n)}, \tau_{n}^{*}, \boldsymbol{\varphi}\right)
\end{aligned}
$$

with

$$
\begin{aligned}
& R_{1}\left(\bar{y}_{n^{\prime}}, \tau, \boldsymbol{\varphi}\right) \\
&= \mathbf{A}(\boldsymbol{\varphi})^{\prime}\left[I-\frac{\mathbf{G}\left(\bar{y}_{n^{\prime}}, \tau ; \boldsymbol{\varphi}\right) \mathbf{u}_{n}(\boldsymbol{\varphi}) \mathbf{1}_{r}^{\prime}}{\mathbf{g}\left(\bar{y}_{n^{\prime}}, \tau ; \boldsymbol{\varphi}\right)^{\prime} \mathbf{u}_{n}(\boldsymbol{\varphi})}\right] \\
& \times \frac{\mathbf{G}\left(\bar{y}_{n^{\prime}}, \tau ; \boldsymbol{\varphi}\right)}{\mathbf{g}\left(\bar{y}_{n^{\prime}}, \tau ; \boldsymbol{\varphi}\right)^{\prime} \mathbf{u}_{n}(\boldsymbol{\varphi})} \\
& R_{2}^{(l)}\left(\bar{y}_{n^{\prime}}, \tau, \boldsymbol{\varphi}\right) \\
&= \mathbf{A}(\boldsymbol{\varphi})^{\prime}\left[I-\frac{\mathbf{G}\left(\bar{y}_{n^{\prime}}, \tau ; \boldsymbol{\varphi}\right) \mathbf{u}_{n}(\boldsymbol{\varphi}) \mathbf{1}_{r}^{\prime}}{\mathbf{g}\left(\bar{y}_{n^{\prime}}, \tau ; \boldsymbol{\varphi}\right)^{\prime} \mathbf{u}_{n}(\boldsymbol{\varphi})}\right] \\
& \times \frac{\left[\partial \mathbf{G}\left(\bar{y}_{n^{\prime}}, \tau ; \boldsymbol{\varphi}\right) / \partial \varphi_{l}\right] \mathbf{u}_{n}(\boldsymbol{\varphi})}{\mathbf{g}\left(\bar{y}_{n^{\prime}}, \tau ; \boldsymbol{\varphi}\right)^{\prime} \mathbf{u}_{n}(\boldsymbol{\varphi})} \\
&+\frac{\left[\partial \mathbf{A}(\boldsymbol{\varphi})^{\prime} / \partial \varphi l\right.}{\mathbf{G}\left(\bar{y}_{n^{\prime}}, \tau ; \boldsymbol{\varphi}\right) \mathbf{u}_{n}(\boldsymbol{\varphi})} \\
& \mathbf{g}\left(\bar{y}_{n^{\prime}}, \tau ; \boldsymbol{\varphi}\right)^{\prime} \mathbf{u}_{n}(\boldsymbol{\varphi})
\end{aligned}
$$

where

$$
\begin{gathered}
\frac{\partial}{\partial \varphi_{l}} \mathbf{G}\left(\bar{y}_{n^{\prime}}, \tau ; \boldsymbol{\varphi}\right)=\frac{\partial}{\partial \varphi_{l}}\left[\mathbf{D}(\tau) \mathbf{B}\left(\bar{y}_{n^{\prime}} ; \boldsymbol{\varphi}\right)\right] \\
=\frac{\partial \mathbf{D}(\tau)}{\partial \varphi_{l}} \mathbf{B}\left(\bar{y}_{n^{\prime}} ; \boldsymbol{\varphi}\right)+\mathbf{D}(\tau) \frac{\partial \mathbf{B}\left(\bar{y}_{n^{\prime}} ; \boldsymbol{\varphi}\right)}{\partial \varphi_{l}}
\end{gathered}
$$


Using these equations, we can recursively calculate $\mathbf{w}_{n}(\varphi)$ at every iteration.

To estimate the set of optimal parameters $\varphi^{*}$, we want to find the maximum of $\ell_{n}(\varphi)$ with respect to $\varphi$, which we will attempt via recursive stochastic approximation. For each parameter $l$ in $\varphi$, at each time $n$, we take $\left(\partial / \partial \varphi_{l}\right)$ of the most recent term inside the summation in Equation (25), to form an incremental score vector

$$
\mathbf{S}\left(\tilde{Y}_{n^{\prime}} ; \boldsymbol{\varphi}\right)=\left(S^{(1)}\left(\tilde{Y}_{n^{\prime}} ; \boldsymbol{\varphi}\right), \ldots, S^{(p)}\left(\tilde{Y}_{n^{\prime}} ; \boldsymbol{\varphi}\right)\right)^{\prime}
$$

with

$$
\begin{aligned}
S^{(l)}\left(\tilde{Y}_{n} ; \boldsymbol{\varphi}\right)= & \frac{\partial}{\partial \varphi_{l}} \log \left[\mathbf{g}\left(\bar{y}_{\xi(n)}, \tau_{n}^{*} ; \boldsymbol{\varphi}\right)^{\prime} \mathbf{u}_{n}(\boldsymbol{\varphi})\right] \\
= & \frac{\mathbf{g}\left(\bar{y}_{\xi(n)}, \tau_{n}^{*} ; \boldsymbol{\varphi}\right)^{\prime}\left[\left(\partial / \partial \varphi_{l}\right) \mathbf{u}_{n}(\boldsymbol{\varphi})\right]}{\mathbf{g}\left(\bar{y}_{\xi(n)}, \tau_{n}^{*} ; \boldsymbol{\varphi}\right)^{\prime} \mathbf{u}_{n}(\boldsymbol{\varphi})} \\
& +\frac{\left[\left(\partial / \partial \varphi_{l}\right) \mathbf{g}\left(\bar{y}_{\xi(n)}, \tau_{n}^{*} ; \boldsymbol{\varphi}\right)\right]^{\prime} \mathbf{u}_{n}(\boldsymbol{\varphi})}{\mathbf{g}\left(\bar{y}_{\xi(n)}, \tau_{n}^{*} ; \boldsymbol{\varphi}\right)^{\prime} \mathbf{u}_{n}(\boldsymbol{\varphi})} \\
= & \frac{\mathbf{g}\left(\bar{y}_{\xi(n)}, \tau_{n}^{*} ; \boldsymbol{\varphi}\right)^{\prime} \mathbf{w}_{n}(\boldsymbol{\varphi})}{\mathbf{g}\left(\bar{y}_{\xi(n)}, \tau_{n}^{*} ; \boldsymbol{\varphi}\right)^{\prime} \mathbf{u}_{n}(\boldsymbol{\varphi})} \\
& +\frac{\left[\left(\partial / \partial \varphi_{l}\right) \mathbf{g}\left(\bar{y}_{\xi(n)}, \tau_{n}^{*} ; \boldsymbol{\varphi}\right)\right]^{\prime} \mathbf{u}_{n}(\boldsymbol{\varphi})}{\mathbf{g}\left(\bar{y}_{\xi(n)}, \tau_{n}^{*} ; \boldsymbol{\varphi}\right)^{\prime} \mathbf{u}_{n}(\boldsymbol{\varphi})}
\end{aligned}
$$

where

$$
\begin{array}{r}
\frac{\partial}{\partial \varphi_{l}} \mathbf{g}\left(\bar{y}_{n^{\prime}}, \tau ; \boldsymbol{\varphi}\right)=\frac{\partial}{\partial \varphi_{l}}\left[\mathbf{d}(\tau) \mathbf{B}\left(\bar{y}_{n^{\prime}} ; \boldsymbol{\varphi}\right)\right] \\
=\frac{\partial \mathbf{d}(\tau)}{\partial \varphi_{l}} \mathbf{B}\left(\bar{y}_{n^{\prime}} ; \boldsymbol{\varphi}\right)+\mathbf{d}(\tau) \frac{\partial \mathbf{B}\left(\bar{y}_{n^{\prime}} ; \boldsymbol{\varphi}\right)}{\partial \varphi_{l}}
\end{array}
$$

and

$$
\tilde{Y}_{n} \triangleq\left(Y_{n}, \mathcal{T}_{n}, \mathbf{u}_{n}(\boldsymbol{\varphi}), \mathbf{w}_{n}(\boldsymbol{\varphi})\right),
$$

with $\left\{\mathcal{T}_{n}\right\}=\left\{\tau_{n}^{*}\right\}$.

The RMLE algorithm takes the form

$$
\boldsymbol{\varphi}_{n+1}=\Pi_{G}\left(\boldsymbol{\varphi}_{n}+\epsilon_{n} \mathbf{S}\left(\tilde{Y}_{n} ; \boldsymbol{\varphi}_{n}\right)\right)
$$

where $\epsilon_{n}$ is a sequence of step sizes satisfying $\epsilon_{n} \geq 0, \epsilon_{n} \rightarrow$ 0 and $\sum_{n} \epsilon_{n}=\infty, G$ is a compact and convex set, and $\Pi_{G}$ is a projection onto set $G$.

Equations (33) and (36) can both be simplified for each type of parameter in $\varphi$. In particular, they must be calculated for the parameters of the chosen duration density $\mathbf{d}(\bar{\tau} ; \boldsymbol{\varphi})$ and observation density $\mathbf{b}(\bar{y} ; \varphi)$.

\section{CONCLUSION}

In this paper, we have presented a derivation of the RMLE algorithm for HSMMs. Simulation results are in progress but are beyond the scope of this paper. We believe that this paper provides a useful tool to researchers studying HSMMs and related algorithms.

\section{APPENDIX}

Here we provide the proof that $\bar{p}_{n^{\prime}}\left(\bar{y}_{1}, \ldots, \bar{y}_{n}, \bar{\tau}_{1}, \ldots, \bar{\tau}_{n^{\prime}} ; \varphi\right)$ is equal to $\prod_{k=1}^{n^{\prime}} \mathbf{d}\left(\bar{\tau}_{k} ; \boldsymbol{\varphi}\right)^{\prime} \mathbf{B}\left(\bar{y}_{k} ; \boldsymbol{\varphi}\right) \overline{\mathbf{u}}_{k}(\boldsymbol{\varphi})$. We have

$$
\begin{aligned}
& \bar{p}_{n^{\prime}}\left(\bar{y}_{1}, \ldots, \bar{y}_{n^{\prime}}, \bar{\tau}_{1}, \ldots, \bar{\tau}_{n^{\prime}}\right) \\
& =p\left(\bar{y}_{1}, \bar{\tau}_{1}\right) p\left(\bar{y}_{2}, \bar{\tau}_{2} \mid \bar{y}_{1}, \bar{\tau}_{1}\right) \cdots \\
& \times p\left(\bar{y}_{n^{\prime}}, \bar{\tau}_{n^{\prime}} \mid \bar{y}_{1}, \ldots, \bar{y}_{n^{\prime}-1}, \bar{\tau}_{1}, \ldots, \bar{\tau}_{n^{\prime}-1}\right) \\
& =\sum_{j} p\left(\bar{y}_{1}, \bar{\tau}_{1}, \bar{x}_{1}=j\right) \sum_{j} p\left(\bar{y}_{2}, \bar{\tau}_{2}, \bar{x}_{2}=j \mid \bar{y}_{1}, \bar{\tau}_{1}\right) \cdots \\
& \times \sum_{j} p\left(\bar{y}_{n^{\prime}}, \bar{\tau}_{n^{\prime}}, \bar{x}_{n^{\prime}}=j \mid \bar{y}_{1}, \ldots, \bar{y}_{n^{\prime}-1}, \bar{\tau}_{1}, \ldots, \bar{\tau}_{n^{\prime}-1}\right) \\
& =\sum_{j} p\left(\bar{y}_{1}, \bar{\tau}_{1} \mid \bar{x}_{1}=j\right) P\left(\bar{x}_{1}=j\right) \\
& \times \sum_{j} p\left(\bar{y}_{2}, \bar{\tau}_{2} \mid \bar{x}_{2}=j\right) P\left(\bar{x}_{2}=j \mid \bar{y}_{1}, \bar{\tau}_{1}\right) \cdots \\
& \times \sum_{j} p\left(\bar{y}_{n^{\prime}}, \bar{\tau}_{n^{\prime}} \mid \bar{x}_{n^{\prime}}=j\right) \\
& \times P\left(\bar{x}_{n^{\prime}}=j \mid \bar{y}_{1}, \ldots, \bar{y}_{n^{\prime}-1}, \bar{\tau}_{1}, \ldots, \bar{\tau}_{n^{\prime}-1}\right) \\
& =\sum_{j} p\left(\bar{y}_{1} \mid \bar{\tau}_{1}, \bar{x}_{1}=j\right) p\left(\bar{\tau}_{1} \mid \bar{x}_{1}=j\right) P\left(\bar{x}_{1}\right) \\
& \times \sum_{j} p\left(\bar{y}_{2} \mid \bar{\tau}_{2}, \bar{x}_{2}=j\right) p\left(\bar{\tau}_{2} \mid \bar{x}_{2}=j\right) P\left(\bar{x}_{2}=j \mid \bar{y}_{1}, \bar{\tau}_{1}\right) \\
& \cdots \times \sum_{j} p\left(\bar{y}_{n^{\prime}} \mid \bar{\tau}_{n^{\prime}}, \bar{x}_{n^{\prime}}=j\right) p\left(\bar{\tau}_{n^{\prime}} \mid \bar{x}_{n^{\prime}}=j\right) \\
& \times P\left(\bar{x}_{n^{\prime}}=j \mid \bar{y}_{1}, \ldots, \bar{y}_{n^{\prime}-1}, \bar{\tau}_{1}, \ldots, \bar{\tau}_{n^{\prime}-1}\right) \\
& =\sum_{j} b_{j}\left(\bar{y}_{1} \mid \bar{\tau}_{1}\right) d_{j}\left(\bar{\tau}_{1}\right) \bar{u}_{1 j} \sum_{j} b_{j}\left(\bar{y}_{2} \mid \bar{\tau}_{2}\right) d_{j}\left(\bar{\tau}_{2}\right) \bar{u}_{2 j} \ldots \\
& \times \sum_{j} b_{j}\left(\bar{y}_{n^{\prime}} \mid \bar{\tau}_{n^{\prime}}\right) d_{j}\left(\bar{\tau}_{n^{\prime}}\right) \bar{u}_{n^{\prime} j} \\
& =\prod_{k^{\prime}=1}^{n^{\prime}} 1_{r}^{\prime} \mathbf{B}\left(\bar{y}_{k^{\prime}} \mid \bar{\tau}_{k^{\prime}}\right) \mathbf{D}\left(\bar{\tau}_{k^{\prime}}\right) \overline{\mathbf{u}}_{k^{\prime}} \\
& =\prod_{k^{\prime}=1}^{n^{\prime}} \mathbf{b}\left(\bar{y}_{k^{\prime}} \mid \bar{\tau}_{k^{\prime}}\right)^{\prime} \mathbf{D}\left(\bar{\tau}_{k^{\prime}}\right) \overline{\mathbf{u}}_{k^{\prime}} \\
& =\prod_{k^{\prime}=1}^{n^{\prime}} \mathbf{d}\left(\bar{\tau}_{k^{\prime}}\right)^{\prime} \mathbf{B}\left(\bar{y}_{k^{\prime}} \mid \bar{\tau}_{k^{\prime}}\right) \overline{\mathbf{u}}_{k^{\prime}} \\
& =\prod_{k^{\prime}=1}^{n^{\prime}} \mathbf{g}\left(\bar{y}_{k^{\prime}}, \bar{\tau}_{k^{\prime}}\right)^{\prime} \overline{\mathbf{u}}_{k^{\prime}}
\end{aligned}
$$

where the third line is due to the Markov assumption, i.e., that each observation and each duration depend only on the current state in an HSMM. Note that if this assumption is 
relaxed, this proof and the RMLE derivation in this paper are no longer valid.

\section{REFERENCES}

[1] François LeGland and Laurent Mével, "Recursive estimation in hidden Markov models," in Proc. 36th IEEE Conf. Decision Contr., San Diego, CA, Dec. 1997.

[2] Vikram Krishnamurthy and George Gang Yin, "Recursive algorithms for estimation of hidden Markov models and autoregressive models with Markov regime," IEEE Trans. Inform. Theory, vol. 48, no. 2, pp. 458-476, Feb. 2002.

[3] John D. Ferguson, "Variable duration models for speech," in Proc. of the Symposium on the Applications of Hidden Markov Models to Text and Speech, John D. Ferguson, Ed., Princeton, NJ, Oct. 1980, IDACRD, pp. 143-179.

[4] M. J. Russell and R. K. Moore, "Explicit modelling of state occupancy in hidden Markov models for automatic speech recognition," in Proc. IEEE Int. Conf. Acoust. Speech Signal Processing, Apr. 1985, vol. 10, pp. 5-8.

[5] S. E. Levinson, "Continuously variable duration hidden Markov models for automatic speech recognition," Computer Speech and Language, vol. 1, pp. 29-45, 1986.

[6] Kevin Murphy, "Hidden semi-Markov models (HSMMs)," http://www.cs.ubc.ca/ murphyk/papers.html, Nov. 2002.

[7] Lawrence R. Rabiner, "A tutorial on hidden Markov models and selected applications in speech recognition," Proc. IEEE, vol. 77, no. 2, pp. 257-286, 1989.
[8] David Kulp, David Haussler, Martin G. Reese, and Frank H. Eeckman, "A generalized hidden Markov model for the recognition of human genes in DNA," in Proc. 4th Int. Conf. Intell. Syst. Molecular Bio., 1996, pp. 134-142.

[9] Martin Russell, "A segmental HMM for speech pattern matching," in Proc. IEEE Int. Conf. Acoust. Speech Signal Processing, 1993, vol. II, pp. 449-502.

[10] Mari Ostendorf, Vassilios V. Digalakis, and Owen A. Kimball, "From HMM's to segment models: A unified view of stochastic modeling for speech recognition," IEEE Trans. Speech Audio Processing, vol. 4, no. 5, pp. 360-378, sep 1996.

[11] Aristotle Arapostathis and Stephen I. Marcus, "Analysis of an identification algorithm arising in the adaptive estimation of Markov chains," Math Control Signals Systems, vol. 3, no. 1, pp. 1-29, 1990.

[12] Vikram Krishnamurthy and John B. Moore, "On-line estimation of hidden Markov model parameters based on the Kullback-Leiber information measure," IEEE Trans. Signal Processing, vol. 41, no. 8, pp. 25572573, Aug. 1993.

[13] Iain B. Collings, Vikram Krishnamurthy, and John B. Moore, "On-line identification of hidden Markov models via recursive prediction error techniques," IEEE Trans. Signal Processing, vol. 42, no. 12, pp. 3535-3539, Dec. 1994.

[14] François LeGland and Laurent Mével, "Recursive identification of HMM's with observations in a finite set," in Proc. 34th IEEE Conf. Decision Contr., New Orleans, Dec. 1995, pp. 216-221.

[15] Tobias Rydén, “On recursive estimation for hidden Markov models," Stochastic Processes and their Applications, vol. 66, pp. 79-96, 1997.

[16] H. Vincent Poor, An Introduction to Signal Detection and Estimation, Springer-Verlag, New York, 1994. 